Dänische Studie

\title{
Senken Statine die Krebssterblichkeit?
}

Krebspatienten, die Statine einnehmen, weisen eine im Schnitt $15 \%$ niedrigere krankheitsspezifische Sterblichkeit auf als Tumorkranke, die keine CholesterinHemmer einnehmen. Die Dosis spielt dabei laut Erkenntnissen dänischer Forscher keine Rolle.

Ärzte der Universität Kopenhagen bedienten sich für ihre Studie des dänischen Melderegisters und zogen daraus die Daten von knapp 296.000 Patienten, die über 40 Jahre alt waren und zwischen 1995 und 2007 eine Krebsdiagnose erhalten hatten. Rund 19.000 hatten schon davor regelmäßig Statine eingenommen, 277.000 waren vor der Diagnose weder mit Statinen noch mit anderen Cholesterin-senkenden $\mathrm{Me}$ dikamenten behandelt worden.
Laut den Berechnungen war die Krebsmortalität der Statin-behandelten Patienten im Follow-up bis Ende 2009 um 15\% geringer als die Malignomsterblichkeit der nicht mit Fettsenkern therapierten Tumorkranken. Die Tagesdosis der eingenommenen Cholesterin-Hemmer schlug sich nicht in der Mortalitätsreduktion nieder. Für 13 Tumorentitäten war die Senkung der Sterblichkeit statistisch signifikant, am stärksten ausgeprägt für das Zervixkarzinom (minus 36\%). Die Mortalität von Lungenkrebspatienten sank um $13 \%$ und die von Kolonkrebskranken um 21\%. Die Sterblichkeit wurde bei Prostatakrebs um 19\%, bei Brustkrebs um $12 \%$ und bei Pankreaskarzinomen um $11 \%$ reduziert.
Die dänischen Forscher halten ihre Ergebnisse für plausibel, weil Statine die Cholesterinsynthese hemmen. Unterbrechungen der entsprechenden Stoffwechselwege in malignen Zellen behindern das Wachstum und die Metastasierung von Krebs. Zudem haben Krebszellen einen hohen Bedarf an extrazellulärem Cholesterin. „Was wir nun brauchen, sind prospektive Untersuchungen der Hypothese, dass die Statineinnahme das Überleben von Krebspatienten verlängert", schreiben die Wissenschaftler.

Robert Bublak

Nielsen SF et al. Statin Use and Reduced CancerRelated Mortality. N Engl J Med. 2012; 367(19): 1792-802.

\section{Späte Diagnose = schlechte Prognose? Nicht unbedingt!}

Französische Onkologen analysierten, wie sich Verzögerungen bei der Diagnose von Krebserkrankungen in der Pädiatrie auswirken - mit teils paradoxen Ergebnissen. Ihre systematische Übersicht zu einer ganzen Reihe pädiatrischer Tumorerkrankungen wie etwa Leukämien, Lymphomen und Hirntumoren zeigte, dass die Zeit bis zur Diagnose wahrscheinlich am meisten von der Tumorbiologie abhängt.

Insgesamt analysierten die Forscher die Daten von 22.619 Patienten aus 98 Studien der vergangenen 40 Jahre. Die Spanne von den ersten Symptomen bis zur Tumordiagnose maß zwischen zwei Wochen und fünf Jahren. Je nach Tumortyp variierte sie stark, wobei im Übersichtszeitraum keine Veränderungen festzustellen waren. Als Maß für die Verzögerung diente der Zeitraum vom ersten Arztkontakt bis zur abschließenden Diagnose.

Negativ wirkte sich in den untersuchten Studien eine Verzögerung bei Retinoblastomen aus, womöglich auch bei Leukämien, Nephroblastomen und Rhabdomyosarkomen (hier reichten die Daten nicht für definitive Schlüsse aus). Keine negativen Folgen hatten späte Diagnosen bei ZNS-Tumoren, Osteosarkomen und
Ewing-Sarkomen. Im Gegenteil: Oft ging eine verspätete Diagnose bei diesen Tumorentitäten mit einer besseren Prognose einher, als es bei einer früheren Identifikation des Leidens der Fall war.

Das erklärt sich den Autoren zufolge aus der Tumorbiologie: schnell wachsende und metastasierende Tumoren verursachen rasche und eindrückliche klinische Zeichen, die dann früh zur Diagnose der meist schon fortgeschrittenen Krebserkrankung führen. Lokal und langsam wachsende Tumoren zeigen oft einen indolenten Verlauf, was trotz späterer Diagnose eine bessere Prognose mit sich bringt. Die Forscher betonen, dass das nicht heiße, dass die Diagnose absichtlich hinausgezögert werden solle.

Robert Bublak

Brasme J-F et al. Delays in diagnosis of paediatric cancers: a systematic review and comparison with expert testimony in lawsuits. Lancet Oncol. 2012;13(10):e445-59.

\section{kurz notiert}

Trastuzumab: Höhere Herzinsuffizienz-Rate bei älteren Brustkrebspatientinnen

Eine Herzinsuffizienz oder Kardiomyopathie tritt bei älteren Brustkrebspatientinnen als Komplikation einer Trastuzumab-Therapie häufiger auf als bisher aus klinischen Studien berichtet wurde. Das ergab eine Auswertung der Daten von über 45.000 Patientinnen im Alter von 67 bis 94 Jahren mit Brustkrebs im Frühstadium [Chen J et al. J Am Coll Cardiol. 2012: doi:10.1016/j.jacc. 2012.07.068]. Die amerikanischen Forscher hatten im Rahmen der Studie verschiedene adjuvante Therapien verglichen. Die angepasste
3-Jahres-Inzidenzrate für eine Herzinsuffizienz oder Kardiomyopathie war bei TrastuzumabBehandlung (32,1/100 Patientinnen) und Anthrazyklin-haltiger Chemotherapie plus Trastuzumab (41,9/100 Patientinnen) höher als bei Patientinnen ohne adjuvante Therapie (18,1/100 Patientinnen; $p<0,001)$. Eine Addition von Trastuzumab zu einer Anthrazyklin-haltigen Chemotherapie erhöhte die Kardiomyopathie-Ereignisse um 12,1, 17,9 und 21,7 pro 100 Patientinnen über den 1-, 2-und 3-Jahres-Follow-up. red 